\title{
Chronically Retained Fecalith following Laparoscopic Appendectomy
}

\author{
Lauren A. Gamble and Andrew Saxe ${ }^{2}$
}

\begin{abstract} following appendectomy. appendectomy.

$\mathbf{I}_{\mathrm{r}}$ NCREASINGLY, appendectomy is performed laparoscopically because of fewer surgical site infections, shorter hospital stays, and decreased post-operative pain compared with open appendectomy [1]. However, this has been accompanied by an increase in intra-abdominal abscesses [1]. One of the leading causes of intra-abdominal abscess following laparoscopic appendectomy is believed to be retained appendicoliths [2]. An appendicolith can serve as a nidus for future abscess, commonly in the pelvis. These usually are recognized within days to months after surgery [3]. The longest interval of an abscess attributable to an appendicolith so far reported is $4 \mathrm{y}$ following laparoscopic appendectomy [4]. We report a case of a patient with peri-hepatic abscess because of retained appendicolith occurring 9 y after laparoscopic appendectomy.
\end{abstract}

Background: Abscess formation because of retained fecalith is a recognized complication of appendectomy. These abscesses may present at a time remote from the appendectomy. No peri-hepatic abscess attributable to a retained fecalith has been reported more than four years following appendectomy.

Methods: A 43-year-old male presented with a subhepatic abscess 9 y following appendectomy. A retained appendicolith was recognized immediately following appendectomy, but was not removed. At his return 9 y after the initial operation, a trial of antibiotic therapy was unsuccessful and he underwent an operation to drain the abscess and retrieve the fecalith. We review the literature pertaining to management of a retained fecalith

Results: Successful management of a subhepatic abscess attributable to a retained fecalith 9 y following

Conclusions: Abscesses attributable to a retained fecalith may present many years following appendectomy. In this setting, antibiotic therapy alone without removal of a retained fecalith is unlikely to be successful.

\section{Case Report}

Clinical history

A 43-year-old male patient who had undergone laparoscopic appendectomy 9 y previously presented to the emergency department (ED) with right-sided abdominal pain spanning from flank to shoulder elicited by lying on his right side. Other symptoms observed upon presentation were chills and sleep disturbances for the previous 2 mo. The appendectomy had been complicated by post-operative respiratory aspiration. A chest computed tomography (CT) demonstrated a retained appendicolith in the right subhepatic space. Because he had other pressing medical issues and the appendicolith was believed to be an incidental finding, the patient was treated with a six-week course of intravenous ertapenem. His medical problems resolved and the appendicolith was left in place.

\section{Physical exam}

Upon presentation $9 \mathrm{y}$ later, the patient had pain on his right side, however, his physical exam was unremarkableneither light or deep abdominal palpation, nor palpation at the right costovertebral angle reproduced right-sided abdominal pain. Murphy's sign was negative. Laboratory findings were significant for elevated C-reactive protein and estimated sedimentation rate. Liver function tests, electrolytes, and complete blood count were within normal limits.

\footnotetext{
${ }^{1}$ College of Human Medicine, ${ }^{2}$ Department of Surgery, College of Human Medicine, Michigan State University, East Lansing, Michigan.

(c) Lauren Gamble and Andrew Saxe 2016; Published by Mary Ann Liebert, Inc. This Open Access article is distributed under the terms of the Creative Commons License (http://creativecommons.org/licenses/by/4.0), which permits unrestricted use, distribution, and reproduction in any medium, provided the original work is properly credited.
} 


\section{Diagnosis}

An abdominal ultrasound revealed a $4.7 \times 3.7 \mathrm{~cm}$ hypoechoic lesion believed to be within the right lobe of the liver. The lesion contained internal echoes and areas of hyper-echogenicity with some through transmission and posterior wall enhancement. A follow-up CT scan of the abdomen and pelvis with intravenous contrast was obtained and further defined the hypo-echoic lesion as a $6.2 \times 4.6 \mathrm{~cm}$ irregularly shaped, rim-enhanced fluid collection within which was a $10 \mathrm{~mm}$ calcified density (see Figs. 1 and 2). The abscess was adjacent to the posterior-superior margin of the liver; the liver itself and gallbladder were unremarkable.

\section{Intervention}

Therapy was initiated with intravenous piperacillintazobactam. The following day an interventional radiologist used a \#5 French sheath needle to aspirate $35 \mathrm{~mL}$ of purulent fluid following which the collection decreased but left the $10 \mathrm{~mm}$ calcification unchanged. The sample contained 40-50 white blood cells per high power field with gram-negative anaerobic rods, which were later identified as Bacteroides fragilis. Intravenous piperacillin-tazobactam was continued for 4 wks after which he was doing well. He and his physicians decided not to remove the appendicolith but rather to reassess in $4 \mathrm{wks}$.

\section{Follow-Up and Results}

Four weeks later, a CT of the abdomen/pelvis demonstrated no change in the appearance of the abscess. He was admitted and underwent hand-assisted laparoscopic abscess drainage. Fifty milliliters of purulent material were drained and the fecalith was removed. A 19-French channel JP drain was placed into the abscess cavity. He was discharged three days later in stable condition to complete a course of intravenous ertapenem.

At the one-month, follow-up appointment the patient complained of abdominal discomfort as well as back pain.

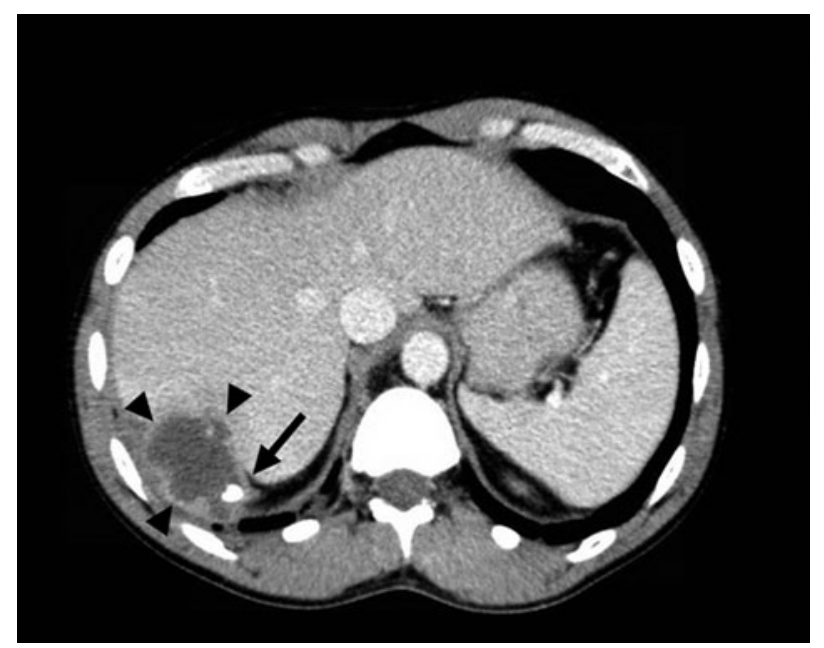

FIG. 1. Computed tomography (CT) with intravenous (IV) contrast of the abdomen in cross-sectional view. Black arrowheads demarcate the fluid-filled lesion to be a $6.2 \times 4.6 \mathrm{~cm}$ with rim enhancement. The black arrow denotes the $10 \mathrm{~mm}$ mural calcification.
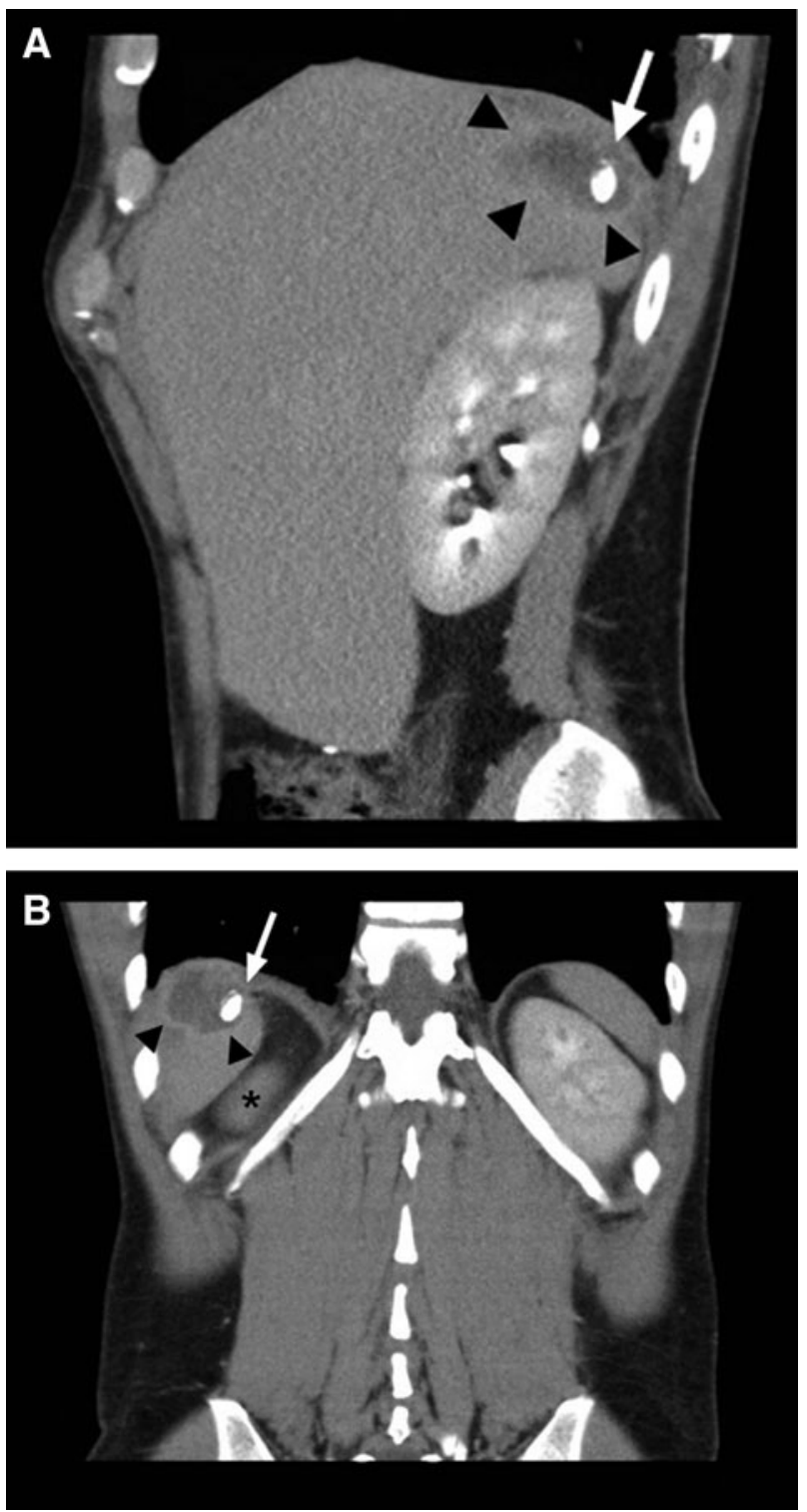

FIG. 2. (A) Computed tomography (CT) with intravenous (IV) contrast of the abdomen in sagittal view. Black arrowheads demarcate the fluid-filled lesion to be a $6.2 \times 4.6 \mathrm{~cm}$ with rim enhancement. The white arrow denotes the $10 \mathrm{~mm}$ mural calcification. (B) Computed tomography (CT) with intravenous (IV) contrast of the abdomen in coronal view. Black arrowheads demarcate the fluid-filled lesion to be a $6.2 \times 4.6 \mathrm{~cm}$ with rim enhancement. The white arrow denotes the $10 \mathrm{~mm}$ mural calcification. The black asterisk denotes the posterior portion of the right kidney.

Magnetic resonance imaging (MRI) of the abdomen demonstrated a residual sub-diaphragmatic fluid collection, similar in size to previous images, as well as phlegmonous change in the right lower quadrant - the fluid collection was unable to be drained by interventional radiology. On the sixth day of admission the patient underwent diagnostic laparoscopy with drainage of the sub-diaphragmatic abscess; the abscess cavity was drained and irrigated with normal saline, there was no evidence of free purulent fluid. Culture of the fluid, again, grew Bacteroides fragilis. He was discharged the next day in stable condition on intravenous ertapenem. 


\section{Discussion}

Most complications arising from appendectomy become apparent within days to months post-operatively. An exception, however, as exemplified by our patient, is an abscess because of a retained appendicolith. Presumably, the peritoneal cavity may not sterilize the foreign body, and when it calcifies its longevity is ensured.

Retained appendicoliths have been associated with serious complications including retro-peritoneal abscess, perihepatic abscess, tubo-ovarian abscess requiring oophorectomy, gluteal region, iliopsoas muscle, and recurrent pelvic abscess [2].

Management of a retained appendicolith is controversial. Most doctors recommend surgical removal of the appendicolith because drainage alone is usually unsuccessful. Buckley et al. reported on five patients who underwent initial CT drainage but all recurred and underwent operations [4]. Several additional reports record the need for surgical removal of the appendicolith to achieve successful outcome $[3,4]$. There have even been reports discussing successful extraction of the appendicolith via percutaneous drainage [2]. Though, arguably, Black et al. provided a case report of an abscess with appendicolith treated with antibiotics alone; length of follow-up was not defined [2]. Singh reported three patients for whom percutaneous drainage without appendicolith retrieval provided definitive treatment [3]. Singh also reported another three patients with post-appendectomy appendicoliths found, incidentally, on CT scans for unrelated issues with none of them requiring intervention [3]. Again, follow-up was not reported [3].

We believe our patient's course supports a recommendation that retained appendicoliths be managed by their removal as soon as is practical after their detection. This recommendation is based upon experiences with patients presenting with complications attributable to retained appendicoliths. We must remember that neither the frequency nor the natural history of retained fecaliths is known.

\section{Author Disclosure Statement}

We have no disclosures of any kind including associations that might create a conflict of interest in connection with this submitted article. There are no competing financial interests for the authors of article.

\section{References}

1. Sauerland S, Lefering R, Holthausen U, et al. Laparoscopic vs conventional appendectomy: A meta-analysis of randomised controlled trials. Langenbeck Arch Surg 1998;383: 289-295.

2. Black MT, Ha BY, Kang YS, et al. Perihepatic abscess caused by dropped appendicoliths following laparoscopic appendectomy: Sonographic findings. J Clin Ultrasound 2013;41:366-369.

3. Singh AK, Hahn PF, Gervais D, et al. Dropped appendicolith: CT findings and implications for management. Am J Roentgenol 2008;190:707-711.

4. Buckley O, Geoghegan T, Ridgeway P, et al. The usefulness of CT guided drainage of abscesses caused by retained appendicoliths. Eur J Radiol 2006;60:80-83.

Address correspondence to: Lauren A. Gamble

Michigan State University

College of Human Medicine

1200 E. Michigan Avenue, Suite 305

Lansing, MI 48912

E-mail: gambleL2@msu.edu

Abbreviations Used
$\mathrm{CT}=$ computed tomography
$\mathrm{ED}=$ emergency department
$\mathrm{IV}=$ intravenous
$\mathrm{MRI}=$ magnetic resonance imaging

Cite this article as: Gamble LA and Saxe A (2016) Chronically retained fecalith following laparoscopic appendectomy, Surgical Infections Case Reports 1:1, 35-37, DOI: 10.1089/crsi.2016.29007.lg. 